\title{
Optical manipulation of microparticles using whispering-gallery modes in a silicon nitride microdisk resonator
}

\author{
Hong Cai and Andrew W. Poon* \\ Photonic Device Laboratory, Department of Electronic and Computer Engineering, The Hong Kong University \\ of Science and Technology, Clear Water Bay, Hong Kong SAR, China \\ *Corresponding author: eeawpoon@ust.hk
}

Received August 18, 2011; revised September 22, 2011; accepted October 3, 2011; posted October 4, 2011 (Doc. ID 153045); published October 28, 2011

\begin{abstract}
We demonstrate optical manipulation of $1 \mu \mathrm{m}$ sized polystyrene microparticles on silicon nitride microdisk resonator devices using whispering-gallery modes in an integrated optofluidic chip. We demonstrate multiple trapping tracks and extended trapping ranges within single wavelengths through exciting high-order modes. We observe various sets of trapping tracks and ranges through exciting various resonance modes. We switch particle traveling tracks by tuning the laser wavelength to various wavelengths. We also observe microparticles assembling along the trapping tracks. (C) 2011 Optical Society of America

OCIS codes: $230.3120,230.5750,170.4520$.
\end{abstract}

Recently, the idea of applying optical tweezers on a chip using the waveguide near field has been gaining increasing interest in optofluidics areas for lab-on-a-chip applications. The use of micro/nanoresonators to obtain enhanced optical fields and functional devices further extends the optical manipulation ability on a chip [1]. Mandal et al. first demonstrated the nano/microparticles manipulation on a silicon photonic crystal resonator [2]. Yang et al. [3] and Lin et al. [4] demonstrated optical switching of the microparticles on SU8 and silicon microring notch filters. Cai et al. demonstrated microparticles add-drop devices using silicon nitride ( $\mathrm{SiN}$ ) microring add-drop filters [5]. Arnold et al. demonstrated nanoparticle manipulation and sizing using whisperinggallery modes (WGMs) on a silica microsphere [ㅁ]

In this Letter, we report optical manipulation of microparticles on a SiN microdisk resonator in an integrated optofluidic chip based on our previous work [7]. By tuning the laser wavelength to excite various microdisk WGMs, we demonstrate particle manipulation on a microdisk resonator with different trapping tracks and extended trapping ranges. We also demonstrate that multiple trapping tracks can be realized using single resonance wavelengths. Multiple particles can be assembled along the trapping tracks on the microdisk resonator.

Figure 1(a) illustrates the principle of optical manipulation of microparticles using a microdisk resonator. Unlike microring resonators which are single-mode devices, microdisk resonators usually support multiple WGMs. High-order WGMs exhibit multiple mode-field maxima (MFM) along the radial direction, which potentially form the multiple particle trapping tracks. The extended mode-field distribution inside the microresonator of the high-order mode extends the particle trapping range inward from the disk edge. The evanescent field outside the microdisk sidewall also enables an additional particle trapping track. Particles trapped by these tracks are driven by the optical force []ㅡ of these resonance traveling waves as demonstrated in microring resonators [므므.
We fabricate $30 \mu \mathrm{m}$ diameter, $700 \mathrm{~nm}$ thick SiN microdisk resonators on top of a $1.8 \mu \mathrm{m}$ silica undercladding layer on a silicon wafer using $i$-line photo-lithography and dry-etching. Figs. 1(b) and 1(c) show the scanning electron microscope (SEM) pictures of the coupling region between the input-coupled waveguide (with a designed width of $0.4 \mu \mathrm{m}$ ) and the microdisk. The coupling gap spacing is $\sim 380 \mathrm{~nm}$. The silica microfluidic channels, which are also fabricated by $i$-line photo-lithography and wet-etching, consist of $6 \mu \mathrm{m}$ height silica walls and a cover glass [5].

We inject diluted colloidal solution of $1 \mu \mathrm{m}$ sized polystyrene particles into the fluidic channel $\left(\sim 16.2 \times 10^{7}\right.$ particles $\left./ \mathrm{ml}\right)$. For spectra measurement, we end-fire $0.5 \mathrm{~mW}$ TM-polarized (E-field $\perp$ chip) laser light around $1550 \mathrm{~nm}$ wavelength into the tapered inputwaveguide using a polarization-maintaining lensed fiber. Figure 2(a) shows the throughput- and drop-spectra of the waveguide-coupled microdisk add-drop device with colloidal solution cladding. The spectra show two free spectral ranges containing multiple WGMs with quality factor $(Q)$ from $\sim 3000$ to $\sim 6000$.

Figure 2(b) shows the 3D finite-element-method (FEMLAB COMSOL) simulated eigen-modes of a $30 \mu \mathrm{m}$ diameter, $700 \mathrm{~nm}$ thick SiN microdisk resonator without coupled waveguides. The calculation assumes an uppercladding of water [refractive index (RI) of 1.32 at $1550 \mathrm{~nm}$
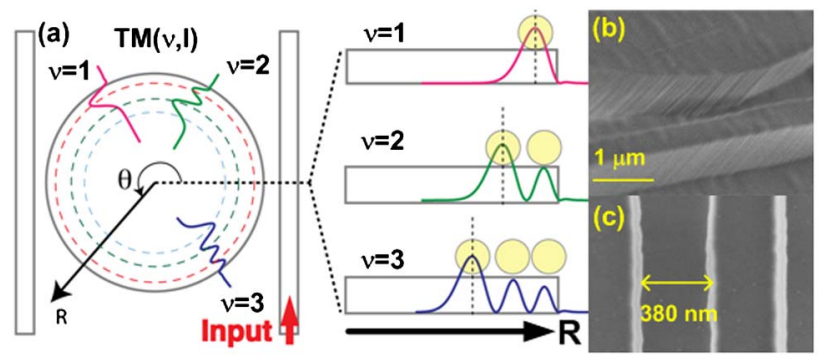

Fig. 1. (Color online) (a) Schematic of particles being trapped by multiple trapping tracks on a microdisk resonator. (b)-(c) SEM pictures of a SiN microdisk resonator: (b) coupling region and (c) coupling gap spacing. 


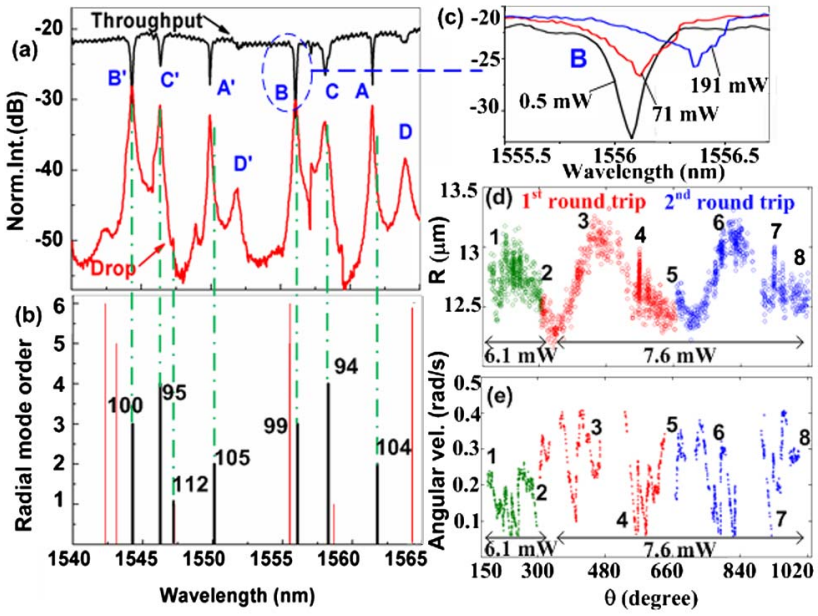

Fig. 2. (Color online) (a) Measured throughput- and dropspectra of the microdisk add-drop device with laser power of $\sim 0.5 \mathrm{~mW}$. (b) Simulated eigen-modes spectrum. The labels are the mode $l$ numbers. The red-thin lines are unidentified modes from the measured spectra. (c) Zoom-in powerdependent throughput-spectrum evolution of resonance $B$. (d) Trajectory (Media 1) and (e) angular velocity of a tworound-trip particle driven at resonance $B$.

range] and a silica layer (RI of 1.44 at $1550 \mathrm{~nm}$ range) as an undercladding. We tune the $\mathrm{RI}$ of $\mathrm{SiN}\left(n_{\mathrm{SiN}}\right)$ to align the calculated mode positions and spacings with those of the measured spectra, assuming the resulted effective RI takes into account the perturbation of the coupled waveguides. The simulation suggests that TM $(\nu, l)$ modes $(\nu$ and $l$ are the radial and azimuthal order numbers) $(2,105),(3,100),(4,95),(2,104),(3,99)$, and $(4,94)$ are aligned with the measured resonance modes A', B', C', $\mathrm{A}, \mathrm{B}$, and $\mathrm{C}$, respectively, using $n_{\mathrm{SiN}}=2.1717 \pm 0.0005$ with $\pm 0.3 \mathrm{~nm}$ tolerance. The comparison between the measured spectra and the simulation suggests the absence of the first-order modes. Considering the surface roughness of the microdisk edge due to the dry-etching process [see Fig. 1(b)], first-order modes which are the closest to the microdisk edge might experience higher scattering loss than higher-order modes. The alignment of the calculated TM $(1,112)$ and the small peak next to resonance C' in the measured drop-spectrum also suggests that first-order modes could be suppressed. The simulation suggests no matching for resonances D' and D.

For particle manipulation, we end-fire erbium-doped fiber amplifier (EDFA) amplified $~ 191 \mathrm{~mW}$ TM-polarized laser light into the input-waveguide. The insertion loss is $\sim 21 \mathrm{~dB}$. We estimate the coupling loss to be $\sim 7 \mathrm{~dB}$ considering the mode area mismatch between the lensed fiber and the tapered waveguide and also the waveguide end-face reflection loss. The estimated power in the input-waveguide is $\sim 7.6 \mathrm{~mW}$ near the microresonator, which is located at $\sim 3.5 \mathrm{~mm}$ from the waveguide input end-face. Figure 2(c) shows the zoom-in throughputspectra at resonance $B$ with spectrum red-shifting and extinction ratio reducing upon increasing the laser power. We attribute this to the SiN absorption-induced thermal-optic effect at $1550 \mathrm{~nm}$ range [9]. The multiple resonance modes upon high-power input are red-shifted to wavelengths A' $(1550.24 \mathrm{~nm})$, B' $(1544.76 \mathrm{~nm})$, C' $(1546.75 \mathrm{~nm}), \quad$ D' $(1552.19 \mathrm{~nm}), \quad$ A $(1561.6 \mathrm{~nm}), \quad$ B
$(1556.46 \mathrm{~nm}), \mathrm{C}(1558.68 \mathrm{~nm})$, and $\mathrm{D}(1564.16 \mathrm{~nm})$. Images of particles are taken by a microscope system with a $50 \times$ objective lens and a charge-coupled device (CCD) camera.

We observe one particle travels two round trips with laser power from $\sim 152 \mathrm{~mW}$ to $\sim 191 \mathrm{~mW}$ upon resonance $B$. Figure 2(d) shows the particle trajectories. The particle is captured from the fluidic medium at laser power of $\sim 152 \mathrm{~mW}$ (estimated input-waveguide power of $\sim 6.1 \mathrm{~mW})$ at position 1 with $(R, \theta)$ of $\left(12.7 \mu \mathrm{m}, 150^{\circ}\right)$. The particle is temporarily stuck at position $2(12.5 \mu \mathrm{m}$, $300^{\circ}$ ) until the laser power is increased to $\sim 191 \mathrm{~mW}$ (estimated input-waveguide power of $\sim 7.6 \mathrm{~mW}$ ). The particle starts the first round trip at position 2 , travels toward position $3\left(13.2 \mu \mathrm{m}, 480^{\circ}\right)$ and finishes the first round trip at position $5\left(12.5 \mu \mathrm{m}, 660^{\circ}\right)$ after $32.6 \mathrm{~s}$. The particle finishes the second round trip at position $8(12.5 \mu \mathrm{m}$, $1020^{\circ}$ ) after $31.5 \mathrm{~s}$. (Media 1) The spikes at positions 4 and 7 in Fig. 2(d) indicate that the particle gets stuck temporarily and suggest that the measurement uncertainty for single-particle is $\Delta R \sim \pm 0.3 \mu \mathrm{m}$ and $\Delta \theta \sim \pm 2^{\circ}$.

Figure 2(e) shows the corresponding angular velocity values of the particle. The oscillation of the velocity is probably affected by the microdisk surface roughness [see Fig. 1(b)] and the fluidic environment. The particle travels at an average angular velocity of $\sim 0.4 \mathrm{rad} / \mathrm{s}$ upon higher laser power $(\sim 191 \mathrm{~mW})$, which is $\sim 0.15 \mathrm{rad} / \mathrm{s}$ faster than that upon the lower laser power $(\sim 152 \mathrm{~mW})$.

Figures 3(a)-3(f) show the radial position $R$ versus angular position $\theta$ plots of accumulated particles trajectories (a total of $N$ single-particle events) upon resonance $\mathrm{A}^{\prime}(N=29), \mathrm{B}^{\prime}(24), \mathrm{C}^{\prime}(23), \mathrm{A}(30), \mathrm{B}(20)$, and $\mathrm{C}$ (22). The particles travel in multiple tracks on the disk (tracks 1 and 2) with $R<15 \mu \mathrm{m}$ and along the microresonator sidewall edge (track 3) with $R>15 \mu \mathrm{m}$ (indicated by the green lines, with the microresonator edge indicated by the red line). Between the trapping tracks, particles cannot be trapped steadily.

Figures $3(\mathrm{~g})-3(\mathrm{i})$ show the simulated cross-section of the resonance mode-field distributions of TM $(2,104)$, $(3,99)$, and $(4,94)$. For mode $(2,104)$, the innermost and the second MFM located at $R \approx 13.6 \mu \mathrm{m}$ and

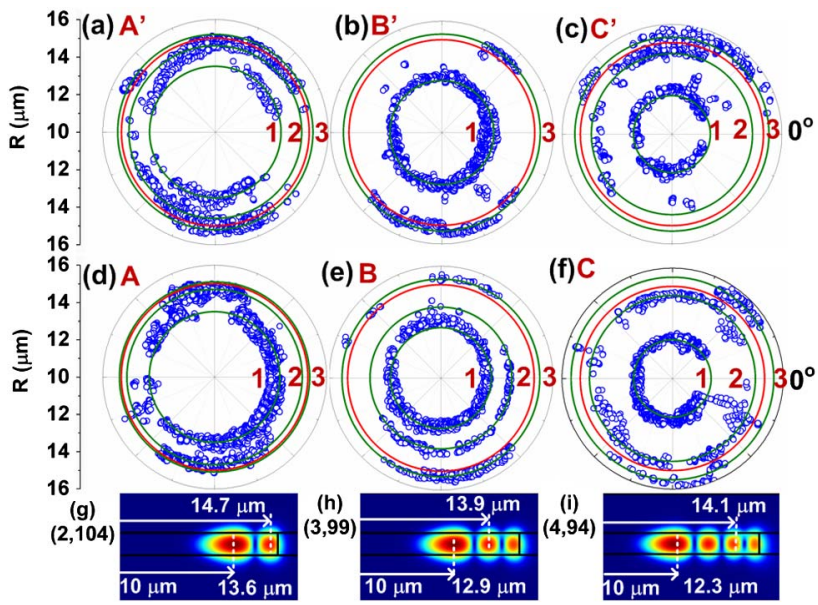

Fig. 3. (Color online) (a)-(f) Measured $R$ versus $\theta$ plots for the resonator-trapped particles upon resonance (a) A', (b) B', (c) C', (d) A, (e) B, and (f) C. (g)-(i) Simulated cross-section of the mode-field distributions of the second-fourth order modes. 
Table 1. Comparison between Various Trapping Tracks and the Simulated Mode-Field Maxima

\begin{tabular}{ccccc}
\hline WGM & Meas. $Q$ & Track1 $(\mu \mathrm{m})$ & Track $2(\mu \mathrm{m})$ & Track 3 $(\mu \mathrm{m})$ \\
\hline A $^{\prime}$ & 6459 & $13.4 \pm 0.2$ & $14.6 \pm 0.2$ & $15.2 \pm 0.1$ \\
A & 6248 & $13.5 \pm 0.2$ & $14.7 \pm 0.2$ & $15.1 \pm 0.1$ \\
$(2,104)$ & - & 13.6 & 14.7 & - \\
B' & 4681 & $12.8 \pm 0.3$ & - & $15.3 \pm 0.1$ \\
B & 3891 & $12.7 \pm 0.2$ & $13.8 \pm 0.1$ & $15.3 \pm 0.1$ \\
$(3,99)$ & - & 12.9 & 13.9 & - \\
C' & 4549 & $12.1 \pm 0.2$ & $14.4 \pm 0.3$ & $15.3 \pm 0.2$ \\
C & 3388 & $12 \pm 0.2$ & $14.5 \pm 0.2$ & $15.5 \pm 0.2$ \\
$(4,104)$ & - & 12.3 & $14.1 / 14.9$ & - \\
\hline
\end{tabular}

$R \approx 14.7 \mu \mathrm{m}$, are consistent with tracks 1 and 2 of resonances A' and A. For mode $(3,99))$, the inner-most MFM $(R \approx 12.9 \mu \mathrm{m})$ is consistent with track 1 for resonances B and B'. The second MFM $(R \approx 13.9 \mu \mathrm{m})$ is consistent with track 2 for resonance $B$. The third MFM cannot be identified in the recorded trajectories, which we attribute to a weak surface field. For mode $(4,94)$, the inner-most MFM $(R \approx 12.3 \mu \mathrm{m})$ is consistent with track 1 for resonance $\mathrm{C}$ and C'. Track 2 locates between third $(R \approx 14.1 \mu \mathrm{m})$ and fourth $(R \approx 14.9 \mu \mathrm{m})$ MFM could be due to the interference between resonance C (or C') and the resonance nearby [see Fig. 2(a)]. Table 1 summarizes the particle trapping tracks, measured $Q \overline{\text { values }}$ and the simulated MFM for the second-fourth order modes.

We switch the particle traveling tracks by tuning the laser wavelength from resonances B-A-C to an offresonance wavelength with laser power of $\sim 265 \mathrm{~mW}$ (estimated input-waveguide power of $\sim 10.3 \mathrm{~mW}$ ). Figure 4(a) shows the optical micrograph of microdisk with particle positions (indicated by the red circles) at various times. Figures 4(b)-4(i) show the zoom-in images of the particle at various times. Upon resonance $B$, the particle is captured from the fluidic medium at $\left(12.6 \mu \mathrm{m}, 0^{\circ}\right)$ [Fig. $\left.4(\mathrm{~b})\right]$ and travels toward $(12.8 \mu \mathrm{m}$, $110^{\circ}$ ) [Fig. 4(c)] with trapping track of $R \sim 12.7 \mu \mathrm{m}$. Upon tuning to resonance $C$, the particle shifts to a track with $R \sim 12.1 \mu \mathrm{m}$ [Fig. $4(\mathrm{~d})$ ] until it reaches $\left(12 \mu \mathrm{m}, 250^{\circ}\right)$ [Fig. 4(e)] where the particle is temporarily stuck (for $66 \mathrm{~s})$. Upon tuning to resonance $A$, the particle shifts its track again $(R \sim 13.5 \mu \mathrm{m})$ [Fig. 4(f)]. When the particle travels back to $\left(13.4 \mu \mathrm{m}, 0^{\circ}\right)$ [Fig. $4(\mathrm{~g})$ ], the laser wavelength is tuned to an off-resonance wavelength at $1563 \mathrm{~nm}$, then the particle is trapped by the inputwaveguide [Fig. 4(h)] and coupled out from the throughput-waveguide [Fig. 4(i)]. Figure 4(j) shows the particle trajectory in an $R$ versus $\theta$ plot with various trapping tracks indicated by the green lines. Figure $4(\mathrm{k})$ shows the particle angular velocity values during the laser wavelength tuning process.

We also observe multiple particles are assembled along the trapping tracks on the microresonator. Upon
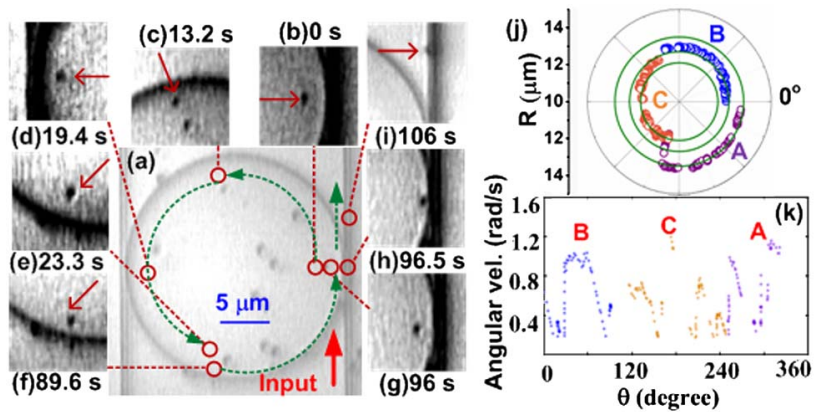

Fig. 4. (Color online) (a) Optical micrograph of the device with particle positions (indicated by the red circles) at various times upon different wavelengths. (b)-(i) Zoom-in images of the particle (labeled by the red arrow) at various times. (j) Measured $R$ versus $\theta$ plot and (k) angular velocity.

laser power of $\sim 191 \mathrm{~mW}$, we record 20,20 , and 13 particles assembling upon resonances $\mathrm{A}, \mathrm{B}$, and $\mathrm{C}$, respectively.

In summary, we demonstrated optical manipulation of microparticles on a SiN microdisk resonator in an integrated optofluidic chip. We observed up to three trapping tracks within one high-order WGM and extended trapping range up to $\sim 3.0 \mu \mathrm{m}$ inward from the resonator edge. We demonstrated various sets of trapping tracks and extended trapping ranges through various high-order WGMs. We also observed that particles can be assembled along the tracking tracks on the microresonator. This multimode resonator with multiple trapping tracks, extended trapping ranges, and potentially being a particle assembler, could be a potential building block for our previously proposed particle-circuits [5] .

This study was substantially supported by a grant from the Research Grants Council of The Hong Kong Special Administrative Region, China, under project no. 618308. The authors acknowledge the HKUST Nanoelectronics Fabrication Facility for fabricating the optofluidic chip.

\section{References}

1. D. Erickson, X. Serey, Y. Chen, and S. Mandal, Lab Chip 11, 995 (2011).

2. S. Mandal, X. Serey, and D. Erickson, Nano Lett. 10, 99 (2010).

3. A. H. Yang and D. Erickson, Lab Chip 10, 769 (2010).

4. S. Lin, E. Schonbrun, and K. Crozier, Nano Lett. 10, 2408 (2010).

5. H. Cai and A. W. Poon, Opt. Lett. 35, 2855 (2010).

6. S. Arnold, D. Keng, S. I. Shopova, S. Holler, W. Zurawsky, and F. Vollmer, Opt. Express 17, 6230 (2009).

7. H. Cai and A. W. Poon, in Proceedings of Conference on Lasers and Electro-Optics (Optical Society of America, 2011), paper JWA110.

8. J. T. Rubin and L. I. Deych, Phys. Rev. A 84, 023844 (2011).

9. K. Wörhoff, P. V. Lambeck, and A. Driessen, J. Lightwave Technol. 17, 1401 (1999). 